\title{
On the Current Error Based Sampled-data Iterative Learning Control with Reduced Memory Capacity
}

\author{
Chiang-Ju Chien ${ }^{1} \quad$ Yu-Chung Hung ${ }^{1}$ Rong-Hu Chi ${ }^{2}$ \\ ${ }^{1}$ Department of Electronic Engineering, Huafan University, New Taipei City 223, Taiwan, China \\ ${ }^{2}$ School of Automation and Electrical Engineering, Qingdao University of Science and Technology, Qingdao 266042, China
}

\begin{abstract}
The design of iterative learning controller (ILC) requires to store the system input, output or control parameters of previous trials for generating the input of the current trial. In order to apply the iterative learning controller for a real application and reduce the memory size for implementation, a current error based sampled-data proportional-derivative (PD) type iterative learning controller is proposed for control systems with initial resetting error, input disturbance and output measurement noise in this paper. The proposed iterative learning controller is simple and effective. The first contribution in this paper is to prove the learning error convergence via a rigorous technical analysis. It is shown that the learning error will converge to a residual set if a forgetting factor is introduced in the controller. All the theoretical results are also shown by computer simulations. The second main contribution is to realize the iterative learning controller by a digital circuit using a field programmable gate array (FPGA) chip applied to repetitive position tracking control of direct current (DC) motors. The feasibility and effectiveness of the proposed current error based sampleddata iterative learning controller are demonstrated by the experiment results. Finally, the relationship between learning performance and design parameters are also discussed extensively.
\end{abstract}

Keywords: Iterative learning control, current error, sampled-data system, memory capacity, field programmable gate array (FPGA) chip.

\section{Introduction}

For repetitive tracking control of industrial applications, iterative learning control (ILC) ${ }^{[1,2]}$ has been known as one of the most effective strategies. To begin with, the proportional-integral-derivative (PID) type iterative learning controllers ${ }^{[3-6]}$ were developed with the control input updated using the data of input and error obtained in the previous iteration. The learning performance is then improved by this simple self-tuning process without using the knowledge of accurate system model. Later, in order to extend the ILC concept to other theoretical researches or practical applications, several ILC frameworks, such as robust $\operatorname{ILC}^{[7,8]}$, adaptive ILC ${ }^{[9-11]}$, adaptive fuzzy $\operatorname{ILC}^{[12]}$ and optimal ILC ${ }^{[13,14]}$ were developed in the past two decades. Most of the ILC algorithms were presented with elegant theoretical analysis. However, some of them required more information of plant's model for the controller design and most of them became more and more complicated. In general, it is hard to implement these ILC algorithms using digital control based approaches for real industrial applications.

Actually, PID-type ILC was widely used in real industrial

\footnotetext{
Regular Paper

Special Issue on Latest Advances in ILC/RLC Theory and Applications

Manuscript received May 1, 2014; accepted October 28, 2014

This work supported by National Science Council, Taiwan, China (No. NSC102-2221-E-211-011) and National Nature Science Foundation of China (No.61374102)

Recommended by Associate Editor Jin-Hua She

(C) Institute of Automation, Chinese Academy of Science and Springer-Verlag Berlin Heidelberg 2015
}

applications because it is simple, effective and easy for realization using any digital controller. In the beginning, most of the PID-type ILCs ${ }^{[3-6]}$ were designed and analyzed in continuous-time domain. Since the ILC is required to store the related control data in memory for generating the control input at the next trial, it is more practical to design and analyze the iterative learning controller in discrete-time domain ${ }^{[15-18]}$ with the controlled plants also being modeled as discrete-time systems. As most of the controlled plants are continuous-time systems, a more interesting issue called sampled-data iterative learning control was studied ${ }^{[19-21]}$. For sampled-data ILC, the plant is modeled as a continuoustime system and the controller is implemented by a discretetime algorithm. Although the technical analysis is similar between pure discrete-time ILC and sampled-data ILC, it is in general more difficult in dealing with the derivation of error equation for convergence analysis of sampled-data ILC.

For realization of ILC algorithms, another interesting issue is the consideration of memory size. For example, it is required to store the control input and tracking error of the previous trial in the memory when implementing a typical PID-type ILC. Suppose the number of data for the desired trajectory is $N$. Then, we need to store $3 N$ data in the controller, i.e., the data of the desired trajectory, control input and tracking error. As the digital integrated circuit (IC) has become a main tool in the implementation of controller nowadays, more memory needed implies more cost of the controller. One of the possible ways to reduce the size of memory is to choose some specified tracking data that are 
desired points at certain time instants ${ }^{[22]}$. Another way is to utilize the technique of terminal ILC where only the terminal point at the end of every trial is used for ILC algorithm $^{[23,24]}$. However, terminal ILC is used for some special processes where only terminal output can be measured. How to reduce the memory size for the case to track the entire desired trajectory is still an interesting issue for ILC design.

To meet the requirements that the iterative learning controller can be implemented by a digital circuit and memory size can be as small as possible, a current error based sampled-data ILC is presented in this paper. Actually, using current error for the design of PID-type ILC is a well known approach in the related area. For example, the ILC which combines feedforward and feedback design can be found in [25-28]. Because the learning error is likely to grow quite significantly before it converges to zero in the process of learning and the rate of convergence is often slow in the pure feedforward iterative learning control, the current error based feedback design is often introduced to improve the learning performance. It is noted that the storage of previous error is still required in this feedbackfeedforward configuration. Some other PID-type ILC designs using only current error can be found in [29, 30]. However, the PID-type ILC proposed in [29] is designed and analyzed in continuous-time domain. In [30], although the PID type ILC is designed in discrete-time domain, a more complicated controller is required to predict the current error at the next time instant. And this introduces some prediction error.

Based on our previous work ${ }^{[31]}$, we investigate the design, analysis and implementation of a simple and practical current error based sampled-data ILC in this paper. The proposed ILC algorithm is easily implemented and will use less memory with only $2 N$ data to be stored if the number of data for desired output trajectory is $N$. The current error based learning mechanism is a simple PD-type algorithm. A forgetting factor is introduced to guarantee the convergence of the learning error. The first main contribution of this paper is to provide a new theoretical analysis for stability and convergence. We show that the tracking error will converge to a residual set even the system has initial resetting error, input disturbance and output measurement noise. The second main contribution is to implement the ILC algorithm by a digital circuit and apply it to repetitive position tracking control of direct current (DC) motors. The digital circuit is realized by using very high speed hardware description language (VHDL) code and then downloaded into a Xilinx field programmable gate array (FPGA) chip. According to the experimental results, we prove that the theoretical derivation is correct and the digital circuit is feasible.

This paper is organized as follows. In Section 2, the problem formulation is given and the current error based sampled-data ILC is proposed. A detailed and rigorous technical proof is shown in Section 3 to guarantee the stability and convergence. A simulation example is provided in Section 4 to demonstrate the correctness of the given theoretical results. In Section 5, the ILC algorithm is implemented by a digital circuit and an experiment is executed for repetitive position tracking control of DC motors. Finally, conclusions are made in Section 6 .

\section{Problem formulation and ILC design}

In this paper, we consider a class of linear systems which can perform a given task repetitively over a finite time interval $[0, T]$ as

$$
\begin{gathered}
\dot{x}_{i}(t)=A x_{i}(t)+B u_{i}(t)+\omega_{i}(t) \\
y_{i}(t)=C x_{i}(t)+\xi_{i}(t)
\end{gathered}
$$

where $x_{i}(t) \in \mathbf{R}^{n \times 1}, y_{i}(t) \in \mathbf{R}^{m \times 1}, u_{i}(t) \in \mathbf{R}^{m \times 1}, \omega_{i}(t) \in$ $\mathbf{R}^{n \times 1}$ and $\xi_{i}(t) \in \mathbf{R}^{m \times 1}$ are state vector, output vector, input vector, input disturbance vector and output measurement noise vector, respectively. Here, $A \in \mathbf{R}^{n \times n}$ is the state matrix, $B \in \mathbf{R}^{n \times m}$ is the input gain matrix, $C \in \mathbf{R}^{n \times m}$ is the output gain matrix, and $i$ denotes the iteration number and $t \in[0, T]$. Let $\Delta T$ be the sampling period of the digital control system with the sampling instant $n=0,1,2, \cdots, N, N \Delta T=T$, where $N$ is a positive integer. The control input $u_{i}(t)$ is assumed to satisfy $u_{i}(t)=u_{i}(n \Delta T)$ for $n \Delta T \leq t<(n+1) \Delta T$. Now, given a realizable desired output $y_{d}(t) \in \mathbf{R}^{m \times 1}$ and an initial state $x_{0}$ which satisfies the following equation without input disturbance and output measurement noise,

$$
\begin{gathered}
\dot{x}_{d}(t)=A x_{d}(t)+B u_{d}(t) \\
y_{d}(t)=C x_{d}(t)
\end{gathered}
$$

where $u_{d}(t)$ is an unknown desired input satisfying $u_{d}(t)=$ $u_{d}(n \Delta T)$ and $n \Delta T \leq t<(n+1) \Delta T$, the control objective is to design a sampled-data ILC $u_{i}(n \Delta T)$ such that the output error at each sampling instant satisfies

$$
\lim _{i \rightarrow \infty}\left\|y_{d}(n \Delta T)-y_{i}(n \Delta T)\right\|<\sigma, \quad \forall n \in\{0,1,2, \cdots, N\}
$$

where $\sigma$ denotes a certain error tolerance bound. The followings are the required assumptions for a realizable controller to achieve the control objective.

Assumption 1. The initial state $x_{i}(0)$, input disturbance $\omega_{i}(t)$ and output measurement noise $\xi_{i}(t)$ satisfy $\left\|x_{d}(0)-x_{i}(0)\right\| \leq \varepsilon_{1},\left\|\omega_{i}(t)\right\| \leq \varepsilon_{2}$ and $\left\|\xi_{i}(t)\right\| \leq \varepsilon_{3}$, for $i=0,1,2, \cdots, \infty, t \in[0, T]$, where $\varepsilon_{1}, \varepsilon_{2}, \varepsilon_{3}$ are positive constants.

Assumption 2. The desired output $y_{d}(n \Delta T)$ and desired input $u_{d}(n \Delta T)$ are bounded with the bound $d$ defined as

$$
d=\sup _{n \in\{0,1,2, \cdots, N\}}\left\|u_{d}(n \Delta T)\right\|
$$

A current error based sampled-data PD-type iterative learning controller is now proposed for system (1) as

$$
\begin{aligned}
& u_{i}(n \Delta T)=(1-\alpha) u_{i-1}(n \Delta T)+\alpha u_{0}(n \Delta T)+ \\
& K_{P} e_{i}(n \Delta T)+K_{D}\left(e_{i}(n \Delta T)-e_{i}((n-1) \Delta T)\right)
\end{aligned}
$$


where $u_{i}(n \Delta T)$ is the iterative learning control input at sampling instant $n \Delta T$ of the $i$-th iteration (current iteration), $u_{i-1}(n \Delta T)$ is the iterative learning control input at sampling instant $n \Delta T$ of the $(i-1)$-th iteration (previous iteration), $e_{i}(n \Delta T)$ is the tracking error at sampling instant $n \Delta T$ of the $i$-th iteration, i.e., $e_{i}(n \Delta T)=y_{d}(n \Delta T)-$ $y_{i}(n \Delta T), n=0,1,2, \cdots, N$. In addition, $0<\alpha<1$ is the forgetting factor, $K_{P}$ and $K_{D}$ are the learning gains of the PD-type learning mechanism and $u_{0}(n \Delta T)$ is the initial bounded learning input. In general, we will set $u_{0}(n \Delta T)$ as close as to $u_{d}(n \Delta T)$ if $u_{d}(n \Delta T)$ can be predicted. Otherwise, we simply set $u_{0}(n \Delta T)=0$ if $u_{d}(n \Delta T)$ is totally unknown.

\section{Analysis of stability and convergence}

Firstly, we define the state error and input error as $\delta x_{i}(t) \equiv x_{d}(t)-x_{i}(t)$ and $\delta u_{i}(t) \equiv u_{d}(t)-u_{i}(t)$. Then, we can derive the following result for $t \in[(n-1) \Delta T, n \Delta T]$

$$
\begin{aligned}
& x_{d}(t)-x_{i}(t)= \\
& x_{d}((n-1) \Delta T)-x_{i}((n-1) \Delta T)+ \\
& \int_{(n-1) \Delta T}^{t}\left(\dot{x}_{d}(\tau)-\dot{x}_{i}(\tau)\right) \mathrm{d} \tau= \\
& \delta x_{i}((n-1) \Delta T)+ \\
& \int_{(n-1) \Delta T}^{t}\left(A \delta x_{i}(\tau)+B \delta u_{i}(\tau)-\omega_{i}(\tau)\right) \mathrm{d} \tau .
\end{aligned}
$$

Since $\delta u_{i}(t)=\delta u_{i}((n-1) \Delta T) t, \quad t \in[(n-1) \Delta T, n \Delta T)$, we have

$$
\begin{aligned}
& \left\|\delta x_{i}(t)\right\| \leq\left\|\delta x_{i}((n-1) \Delta T)\right\|+ \\
& \int_{(n-1) \Delta T}^{t}\left(a\left\|\delta x_{i}(\tau)\right\|+b\left\|\delta u_{i}(\tau)\right\|+\varepsilon_{2}\right) \mathrm{d} \tau \leq \\
& \left\|\delta x_{i}((n-1) \Delta T)\right\|+a \int_{(n-1) \Delta T}^{t}\left\|\delta x_{i}(\tau)\right\| \mathrm{d} \tau+ \\
& b \Delta T\left\|\delta u_{i}((n-1) \Delta T)\right\|+\varepsilon_{2} \Delta T
\end{aligned}
$$

where $\|A\|=a$ and $\|B\|=b$. By using Bellman-Gronwall Lemma, (4) can be rewritten as

$$
\begin{aligned}
& \left\|\delta x_{i}(t)\right\| \leq \\
& \quad \mathrm{e}^{a t}\left(\left\|\delta x_{i}((n-1) \Delta T)\right\|+\right. \\
& \left.b \Delta T\left\|\delta u_{i}((n-1) \Delta T)\right\|+\varepsilon_{2} \Delta T\right) \leq \\
& \quad a_{1}\left\|\delta x_{i}((n-1) \Delta T)\right\|+ \\
& \quad a_{2} \Delta T\left\|\delta u_{i}((n-1) \Delta T)\right\|+a_{1} \varepsilon_{2} \Delta T
\end{aligned}
$$

where $a_{1}=\mathrm{e}^{a T}$. Finally, let the time $t=n \Delta T$, we have

$$
\begin{aligned}
& \left\|\delta x_{i}(n \Delta T)\right\| \leq \\
& \quad a_{1}\left\|\delta x_{i}((n-1) \Delta T)\right\|+a_{2} \Delta T\left\|\delta u_{i}((n-1) \Delta T)\right\|+ \\
& \quad a_{1} \varepsilon_{2} \Delta T .
\end{aligned}
$$

Solving the difference equation (6) iteratively, we can get

$$
\begin{aligned}
& \left\|\delta x_{i}(n \Delta T)\right\| \leq \\
& a_{1}^{n}\left\|\delta x_{i}(0)\right\|+\sum_{j=0}^{n-1} a_{1}^{n-1-j} a_{2} \Delta T\left\|\delta u_{i}(j \Delta T)\right\|+ \\
& \sum_{j=0}^{n-1} a_{1}^{n-j} \varepsilon_{2} \Delta T .
\end{aligned}
$$

Now, we state the main result in the following theorem.

Theorem 1. Let the system (1) satisfy Assumption 1 and the desired input as well as desired output satisfy Assumption 2. If we apply the current error based sampleddata iterative learning controller (3) to system (1), then we can guarantee that

$$
\lim _{i \rightarrow \infty}\left\|y_{d}(n \Delta T)-y_{i}(n \Delta T)\right\| \leq \sigma, \quad \forall n \in\{0,1, \cdots, N\}
$$

for some positive constant $\sigma$ depending on $\varepsilon_{1}, \varepsilon_{2}, \varepsilon_{3}$ and $\alpha$.

Proof. To simplify the analysis, we use $n$ to replace $n \Delta T$ in the following derivation. First of all, we derive the equation for output error as

$$
\begin{aligned}
& e_{i}(n)=y_{d}(n)-y_{i}(n)-\xi_{i}(n)= \\
& y_{d}(n)-y_{i}(n-1)+\int_{n-1}^{n}\left(\dot{y}_{\mathrm{d}}(\tau)-\dot{y}_{i}(\tau)\right) \mathrm{d} \tau-\xi_{i}(n)= \\
& e_{i}(n-1)+C \int_{n-1}^{n}\left(A\left(x_{d}(\tau)-x_{i}(\tau)\right)+\right. \\
& \left.B\left(u_{d}(\tau)-u_{i}(\tau)\right)-\omega_{i}(\tau)\right) \mathrm{d} \tau-\xi_{i}(n)= \\
& e_{i}(n-1)+C \int_{n-1}^{n}\left(A \delta x_{i}(\tau)-\omega_{i}(\tau)\right) \mathrm{d} t+ \\
& C B \Delta T \delta u_{i}(n-1)-\xi_{i}(n) .
\end{aligned}
$$

The input error $\delta u_{i}(n)=u_{d}(n)-u_{i}(n)$ is derived by using (8) as

$$
\begin{aligned}
& \delta u_{i}(n)=u_{d}(n)-(1-\alpha) u_{i-1}(n)-\alpha u_{0}(n)- \\
& K_{P} e_{i}(n)-K_{D}\left(e_{i}(n)-e_{i}(n-1)\right)= \\
& (1-\alpha) \delta u_{i-1}(n)-K_{P} e_{i}(n)- \\
& K_{D}\left(e_{i}(n)-e_{i}(n-1)\right)+\alpha \delta u_{0}(n)= \\
& (1-\alpha) \delta u_{i-1}(n)-K_{P} C \delta x_{i}(n)-K_{P} C \xi_{i}(n)- \\
& K_{D} C \int_{n-1}^{n}\left(A \delta x_{i}(\tau)-\omega_{i}(\tau)\right) \mathrm{d} \tau- \\
& K_{D} C B \Delta T \delta u_{i}(n-1)+K_{D} \xi_{i}(n)+\alpha \delta u_{0}(n) .
\end{aligned}
$$

Let $\left|K_{P}\right|=k_{P}$ and $\left|K_{D}\right|=k_{d}$. Taking norms on (9) and 
using (5), we have

$$
\begin{aligned}
& \left\|\delta u_{i}(n)\right\| \leq \\
& \quad(1-\alpha)\left\|\delta u_{i-1}(n)\right\|+k_{p}\left\|\delta x_{i}(n)\right\|+k_{p} c \varepsilon_{3}+ \\
& \quad k_{d} c a \int_{n-1}^{n}\left\|\delta x_{i}(\tau)\right\| \mathrm{d} \tau+k_{d} c \varepsilon_{2} \Delta T+ \\
& \quad k_{d} c b \Delta T\left\|\delta u_{i}(n-1)\right\|+k_{d} \varepsilon_{3}+\alpha\left\|\delta u_{0}(n)\right\| \leq \\
& \quad(1-\alpha)\left\|\delta u_{i-1}(n)\right\|+k_{p}\left\|\delta x_{i}(n)\right\|+k_{p} c \varepsilon_{3}+ \\
& \quad k_{d} c a \Delta T\left(a_{1}\left\|\delta x_{i}(n-1)\right\|+a_{2} \Delta T\left\|\delta u_{i}(n-1)\right\|+\right. \\
& \left.a_{1} \varepsilon_{2} \Delta T\right)+k_{d} c \varepsilon_{2} \Delta T+k_{d} c b \Delta T\left\|\delta u_{i}(n-1)\right\|+ \\
& \quad k_{d} \varepsilon_{3}+\alpha\left\|\delta u_{0}(n)\right\| \leq(1-\alpha)\left\|\delta u_{i-1}(n)\right\|+ \\
& k_{1}\left\|\delta x_{i}(n)\right\|+k_{2}\left\|\delta x_{i}(n-1)\right\|+k_{3}\left\|\delta u_{i}(n-1)\right\|+k_{4}
\end{aligned}
$$

where $k_{1}, k_{2}, k_{3}, k_{4}$ are suitably defined positive constants and $k_{4}$ is a constant depending on $\Delta T, \varepsilon_{2}, \varepsilon_{3}$ and $\alpha$. By using the result of $(7),(10)$ can be rewritten as

$$
\left\|\delta u_{i}(n)\right\| \leq(1-\alpha)\left\|\delta u_{i-1}(n)\right\|+\beta \sum_{j=0}^{n-1}\left\|\delta u_{i}(j)\right\|+\gamma
$$

where $\beta$ and $\gamma$ are some suitably defined positive constants and $\gamma$ is a constant depending on $\Delta T, \varepsilon_{1} \varepsilon_{2}, \varepsilon_{3}$ and $\alpha$. It is noted that $\gamma=0$ if $\varepsilon_{1}=\varepsilon_{2}=\varepsilon_{3}=\alpha=0$. Now, we expand (11) for $n=0,1,2, \cdots, N$ as

$$
\begin{aligned}
& \left\|\delta u_{i}(0)\right\| \leq(1-\alpha)\left\|\delta u_{i-1}(0)\right\|+\gamma, \\
& \left\|\delta u_{i}(1)\right\|-\beta\left\|\delta u_{i}(0)\right\| \leq(1-\alpha)\left\|\delta u_{i-1}(1)\right\|+\gamma, \\
& \left\|\delta u_{i}(2)\right\|-\beta\left\|\delta u_{i}(1)\right\|-\beta\left\|\delta u_{i}(0)\right\| \leq \\
& (1-\alpha)\left\|\delta u_{i-1}(2)\right\|+\gamma \\
& \cdots \\
& \left\|\delta u_{i}(N)\right\|-\beta\left\|\delta u_{i}(N-1)\right\| \cdots-\beta\left\|\delta u_{i}(0)\right\| \leq \\
& (1-\alpha)\left\|\delta u_{i-1}(N)\right\|+\gamma .
\end{aligned}
$$

The above inequalities can be rearranged into the matrix form as

$$
\begin{gathered}
{\left[\begin{array}{cccc}
1 & 0 & \cdots & 0 \\
-\beta & 1 & \ddots & 0 \\
\vdots & \ddots & \ddots & \vdots \\
-\beta & \cdots & -\beta & 1
\end{array}\right]\left[\begin{array}{c}
\left\|\delta u_{i}(0)\right\| \\
\left\|\delta u_{i}(1)\right\| \\
\vdots \\
\left\|\delta u_{i}(N)\right\|
\end{array}\right] \leq} \\
(1-\alpha)\left[\begin{array}{c}
\left.\| \delta u_{i-1}^{(} 0\right) \| \\
\left.\delta u_{i-1}^{(} 1\right) \| \\
\vdots \\
\left.\| \delta u_{i-1}^{(} N\right) \|
\end{array}\right]+\gamma\left[\begin{array}{c}
1 \\
1 \\
\vdots \\
1
\end{array}\right] .
\end{gathered}
$$

Define $\delta U_{i}=\left[\left\|\delta u_{i}(0)\right\|, \cdots,\left\|\delta u_{i}(N)\right\|\right]^{\mathrm{T}}, I_{1}=[1, \cdots, 1]^{\mathrm{T}}$ and note that

$$
\begin{gathered}
{\left[\begin{array}{llll}
1 & 0 & \cdots & 0 \\
-\beta & 1 & \ddots & 0 \\
\vdots & \ddots & \ddots & \vdots \\
-\beta & \cdots & -\beta & 1
\end{array}\right]^{-1}=} \\
\\
{\left[\begin{array}{ccccc}
1 & & 0 & \cdots & 0 \\
\beta & & 1 & \ddots & 0 \\
\vdots & & \ddots & \ddots & \vdots \\
\beta(\beta+1)^{N-1} & \cdots & \beta & 1
\end{array}\right] \equiv \Lambda .}
\end{gathered}
$$

We can now rewrite (12) in the matrix form as

$$
\delta U_{i} \leq(1-\alpha) \Lambda \delta U_{i-1}+\gamma \Lambda I_{1} .
$$

The solution of (13) can be easily derived as

$$
\delta U_{i} \leq((1-\alpha) \Lambda)^{i} \delta U_{0}+\sum_{j=0}^{i-1} \gamma((1-\alpha) \Lambda)^{j} \Lambda I_{1} .
$$

Since matrix $(1-\alpha) \Lambda$ is a triangular matrix and all the eigenvalues are less than 1 , we conclude that

$$
\lim _{i \rightarrow \infty} \delta U_{i} \leq \lim _{i \rightarrow \infty} \sum_{j=0}^{i-1} \gamma((1-\alpha) \Lambda)^{j} \Lambda I_{1} .
$$

This implies that the input error $\delta u_{i}(n)$ is bounded, $\forall i \geq 1$ and $\forall n \in\{0,1,2, \cdots, N\}$. Furthermore, the norm of input error will converge to a residual set whose level of magnitude depends on the uncertainty bounds $\varepsilon_{1}, \varepsilon_{2}, \varepsilon_{3}$ and the value of forgetting factor $\alpha$

Based on the result of (15), we have

$$
\lim _{i \rightarrow \infty}\left\|\delta u_{i}(n)\right\|=\lim _{i \rightarrow \infty}\left\|u_{d}(n)-u_{i}(n)\right\| \leq \gamma h
$$

for some $h>0$. Furthermore, according to (7), we can find $h_{1}, h_{2}>0$ such that

$$
\lim _{i \rightarrow \infty}\left\|\delta x_{i}(n)\right\| \leq \gamma h_{1}+h_{2}\left(\varepsilon_{1}+\varepsilon_{2}\right) .
$$

This implies that

$$
\begin{aligned}
& \lim _{i \rightarrow \infty}\left\|y_{d}(n)-y_{i}(n)\right\| \leq \\
& \lim _{i \rightarrow \infty}\left\|C x_{d}(n)-C x_{i}(n)-\xi_{i}(n)\right\| \leq \\
& c \gamma h_{1}+c h_{2}\left(\varepsilon_{1}+\varepsilon_{2}\right)+\varepsilon_{3} \equiv \sigma .
\end{aligned}
$$

Remark 1. It is noted that the converged tracking error will only depend on $\alpha$ only if $\varepsilon_{1}=\varepsilon_{2}=\varepsilon_{3}=0$ In this case, $\sigma$ in (16) will be smaller if the value of $\alpha$ is smaller from theoretical point of view. We will demonstrate this observation via some experimental results.

Remark 2. In Theorem 1, the convergence is ensured by the existence of the forgetting factor $\alpha$. However, the choice of the PD gains is also important for this ILC algorithm. Actually, the PD learning mechanism plays a role of feedback controller if iterative learning process is not executed. 
The choice of PD learning gains should stabilize the closed loop system in order to compensate for the output error to a small region. Then, the iterative learning controller can drive the output error to a more reasonable value around zero.

\section{Simulation example}

In this section, we use the following second order system for numerical simulation

$$
\begin{aligned}
& \dot{x}_{i}(t)=\left[\begin{array}{cc}
0 & 1 \\
1 & -4
\end{array}\right] x_{i}(t)+\left[\begin{array}{l}
0 \\
1
\end{array}\right] u_{i}(t)+\left[\begin{array}{c}
0 \\
0.01
\end{array}\right] \operatorname{randn}(t) \\
& y_{i}(t)=\left[\begin{array}{ll}
0 & 1
\end{array}\right] x_{i}(t)+0.01 \operatorname{randn}(t)
\end{aligned}
$$

where $\operatorname{randn}(t)$ is a generator of random number with normal distribution, i.e., mean $=0$ and variance $=1$. The sampling period and desired trajectory are chosen as $0.01 \mathrm{~s}$ and $y_{d}(t)=5 t(2-t), t \in[0,1]$. We assume that the initial output at each iteration satisfies

$$
y_{i}(0)=x_{i, 2}(0)+0.01 \operatorname{randn}(0) \equiv 0.1+0.01 \operatorname{randn}(0) .
$$

This implies that there will exist an initial output error at the beginning of each trial. The current error based sampled-data PD-type ILC is given as

$$
\begin{aligned}
& u_{i}(n \Delta T)=(1-\alpha) u_{i-1}(n \Delta T)+ \\
& \quad K_{p} e_{i}(n \Delta T)+K_{D}\left(e_{i}(n \Delta T)-e_{i}((n-1) \Delta T)\right) .
\end{aligned}
$$

It is noted that we set $u_{0}(n \Delta T)=0$ since we assume that we don't have any prior information about the ideal desired input $u_{d}(n \Delta T)$. The current error based PD-type ILC is designed with learning gains as $K_{P}=5, K_{D}=5$ and forgetting factor $\alpha=0.01$. In order to make some comparisons between the traditional previous error based sampled-data PD-type ILC, we use the following algorithm for another simulation,

$$
\begin{aligned}
u_{i}(n \Delta T)= & u_{i-1}(n \Delta T)+K_{P 1} e_{i-1}(n \Delta T)+ \\
& K_{D 1}\left(e_{i-1}((n+1) \Delta T)-e_{i-1}(n \Delta T)\right)
\end{aligned}
$$

with $K_{P 1}=2$ and $K_{D 1}=10$. Furthermore, a sampleddata PD-type ILC using both previous error and current error design is also given as

$$
\begin{aligned}
& u_{i}(n \Delta T)= \\
& u_{i-1}(n \Delta T)+K_{P 1} e_{i-1}(n \Delta T)+K_{D 1}\left(e_{i-1}((n+1) \Delta T)-\right. \\
& \left.e_{i-1}(n \Delta T)\right)+K_{P 2} e_{i}(n \Delta T)+K_{D 2}\left(e_{i}(n \Delta T)-\right. \\
& \left.e_{i}((n-1) \Delta T)\right)
\end{aligned}
$$

with $K_{P 1}=2, K_{D 1}=10, K_{P 2}=5$ and $K_{D 2}=5$. It is noted that the previous error based PD gains of (19) are the same as those in (18), and the current error based PD gains of (19) are the same as those in (17). The maximum absolute output error is defined as

$$
e_{i, \max }=\max _{n \in\{1,2, \cdots, 100\}}\left|y_{d}(n \Delta T)-y_{i}(n \Delta T)\right| .
$$

In Fig. 1, we show the maximum absolute output error versus the iteration number. The solid line, dashed line and dotted line are the simulation results using ILC algorithms of (17), (18) and (19) respectively. It is clear that the previous error based sampled-data PD-type ILC can ensure the learning error convergence (dashed line) if the PD learning gains are suitably chosen. The learning performance can be further improved (dotted line) if the current error based PD-type ILC is added. This implies that the learning performance is in general better for ILC algorithm using both previous error and current error design. However, it is interesting to note that the ILC algorithm using only current error design (17) can almost have the same performance (solid line) as that obtained by (19).

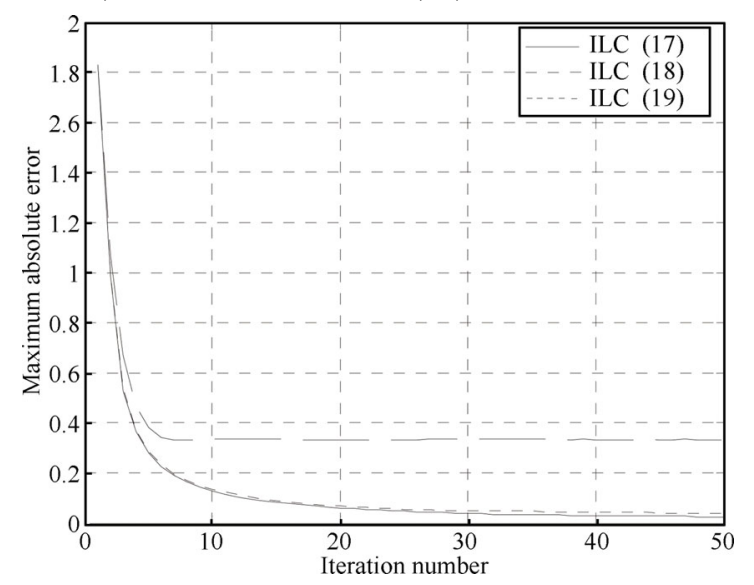

Fig. 1 Maximum absolute output error $e_{i, \max }$ versus the iteration number $i$, solid line for ILC (17), dashed line ILC (18) and dotted line for ILC (19)

In order to study the relationship between forgetting factor and residual learning error as proved in Theorem 1, we use the current error based sampled-data PDtype ILC (17) for simulation with three different values of $\alpha$. Fig. 2 shows the behavior of error convergence for $\alpha=0.1,0.01$ and 0.001 , respectively. In general, the residual learning error will be small if $\alpha$ is small. But there is no significant difference if $\alpha$ is small enough.

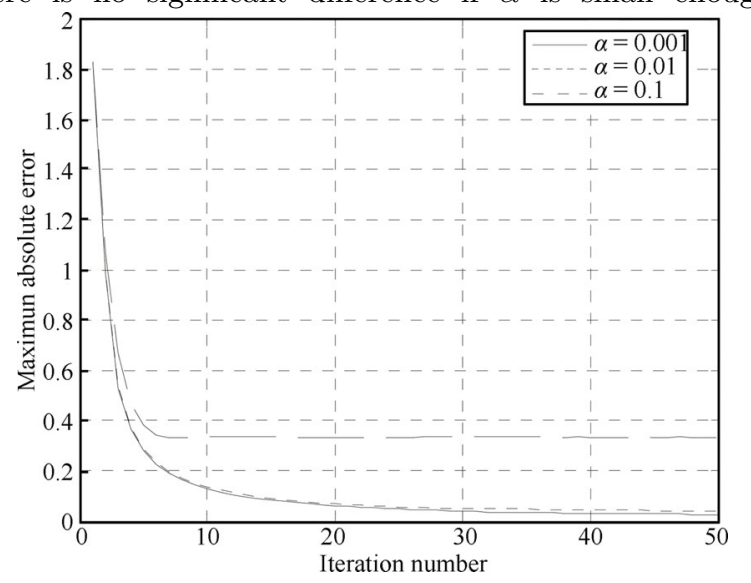

Fig. 2 Maximum absolute output error $e_{i, \max }$ versus the iteration number $i$, solid line for $\alpha=0.001$, dotted line for $\alpha=0.01$, dashed line for $\alpha=0.1$ 


\section{Application to position tracking con- trol of DC motors}

In order to demonstrate the feasibility and effectiveness of the proposed current error based sampled-data iterative learning controller, we apply this controller to execute a repetitive position tracking control task of DC motor. The iterative learning controller is implemented by a digital circuit using VHDL code. The circuit code is compiled by Xilinx ISE and then downloaded into a Xilinx FPGA chip. Fig. 3 shows the hardware setup for the experiment in this paper. The experiment system includes a DC motor, an FPGA control board, a digital input output (DIO) interface card and a personal computer (PC). The experimental data is transmitted to PC by the FPGA control board through the DIO interface card. Then a Matlab program in PC will store the data and show the output position trajectory.

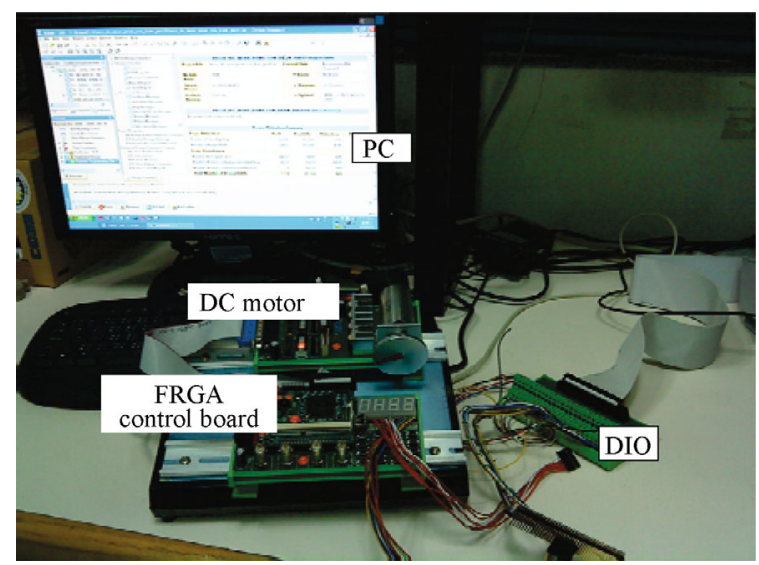

Fig. 3 The hardware setup for experiment

In this experiment, the desired position trajectory is given as that in Fig. 4. The desired position trajectory is designed to rotate the motor clockwise from an initial position of $0^{\circ}$ to a final position of $90^{\circ}$ for $2 \mathrm{~s}$ and then rotate the motor anticlockwise to the initial position for another $2 \mathrm{~s}$. In other word, the period of one iteration is $4 \mathrm{~s}$. The sampling period $\Delta T$ is set to be $0.02 \mathrm{~s}$ so that there are 200 sampling data in one iteration. Due to the current error based design, only 400 registers instead of 600 registers are required for the memory to store the required data.

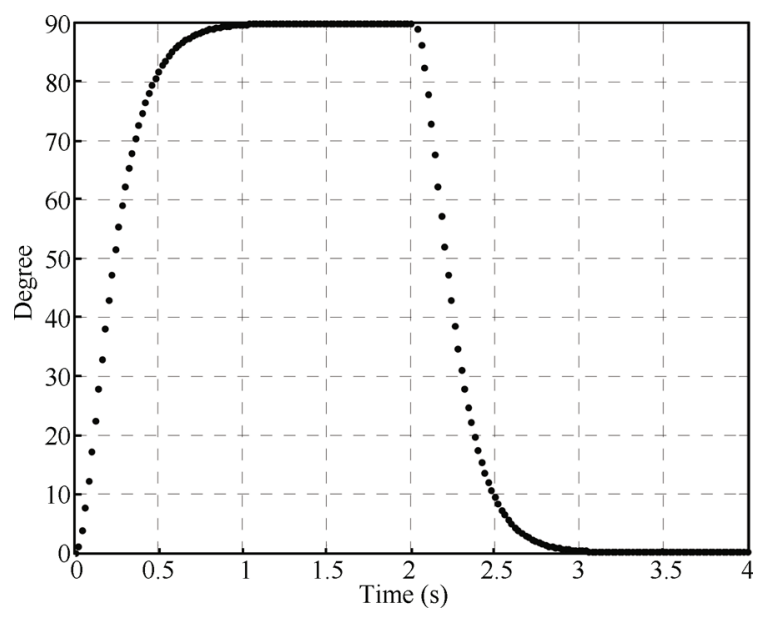

Fig. 4 Desired position trajectory for one iteration

The block diagram of the experiment system is shown in Fig. 5. The current error based sampled-data PD-type ILC (17) is realized with learning gains of $K_{P}=0.5$ and $K_{D}=0.5$. The proposed iterative learning control circuit includes a memory (register) which stores the input signal of the previous iteration and a current error learning mechanism circuit which generates the equation of the algorithm $K_{P} e_{i}(n \Delta T)+K_{D}\left(e_{i}(n \Delta T)-e_{i}((n-1) \Delta T)\right)$. Another memory is utilized to store the initial input $u_{0}(n \Delta T)$ if required. However, it is omitted in this experiment. In Fig. 5, main control circuit is designed to control the circuit timing, to generate the pulse width modulation (PWM) signal, to decode the motor position signal $Y$ and to serve as the I/O interface between the control circuit and DC motor or PC.

In Fig. 6 (a), we show the experiment of motor position trajectory at the first iteration using

$u_{1}(n \Delta T)=K_{P} e_{1}(n \Delta T)+K_{D}\left(e_{1}(n \Delta T)-e_{1}((n-1) \Delta T)\right)$.

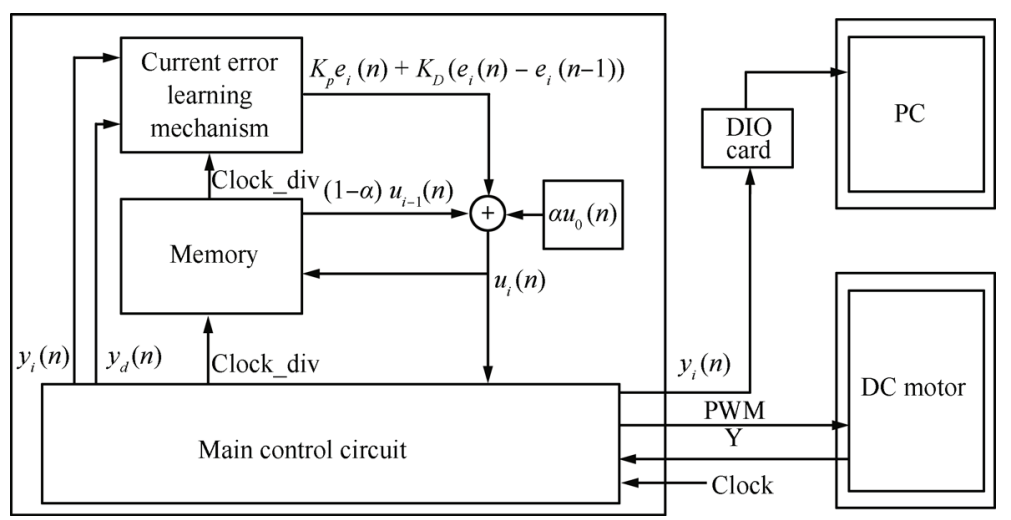

Fig. 5 Block diagram of the experiment system 
In other word, only the PD-type feedback controller is used for the initial trial. Even though we assume that the dynamic model of the DC motor is unknown before controller design, we can still easily find suitable PD gains of $K_{P}=0.5$ and $K_{D}=0.5$ such that the closed loop is stable and the output tracking performance is acceptable. However, there exists an apparent steady state error which is about $1.6199^{\circ}$. Next, we show the experiment of motor position trajectories at the 5th iteration and 10th iteration respectively in Figs. 6 (b) and (c) with $\alpha=0.1$. It can be found from the experimental results that the tracking performance is improved under the proposed iterative learning controller since the steady state errors are reduced to be $0.54^{\circ}$ in Fig. 6 (b) and $0.0899^{\circ}$ in Fig. 6 (c), respectively. Furthermore, the root mean square (RMS) values of learning errors at the 1 st, 5 th and 10 th iteration are 0.7156 , 0.3057 and 0.2862 , respectively. Based on these results, we can conclude the effectiveness and feasibility of the proposed current error based sampled-data PD-type iterative learning controller.

In addition to the results given in Fig. 6, several experiments were also conducted for the study of relationship between the value of $\alpha$ and the learning performance. Under the same learning gains of $K_{P}=0.5$ and $K_{D}=0.5$, we choose three different values of $\alpha$ and repeat the experiments for 10 iterations. The RMS values of the learning errors at the 10th iteration for the three experiments are $0.2862(\alpha=0.1), 0.4017(\alpha=0.2)$ and $0.5698(\alpha=0.4)$, respectively. In general, the RMS value of learning error decreases and the rate of convergence is a little faster if $\alpha$ is smaller.

\section{Conclusions}

In this paper, we propose a current error based sampleddata iterative learning controller and give a theoretical analysis as well as a real application. A PD-type learning mechanism is designed by using current error data so that the memory size can be reduced. A new theoretical proof is given to show the convergence of the learning control system. The PD-type learning mechanism which can be treated as a feedback controller compensates for the output error to a small region so that the iterative learning controller can drive the output error further to a more reasonable value around zero. In order to demonstrate the feasibility and effectiveness, some simulation results are presented. Furthermore, the proposed iterative learning controller is also implemented by a digital circuit for experiment. We use VHDL as a design tool to realize the digital circuit and download the code into an FPGA chip as the controller for repetitive position tracking control of DC motors. According to the simulation and experimental results, we prove that the theoretical analysis is correct and the corresponding digital circuit is feasible.

A future work is to extend the proposed design to more general class of nonlinear systems.

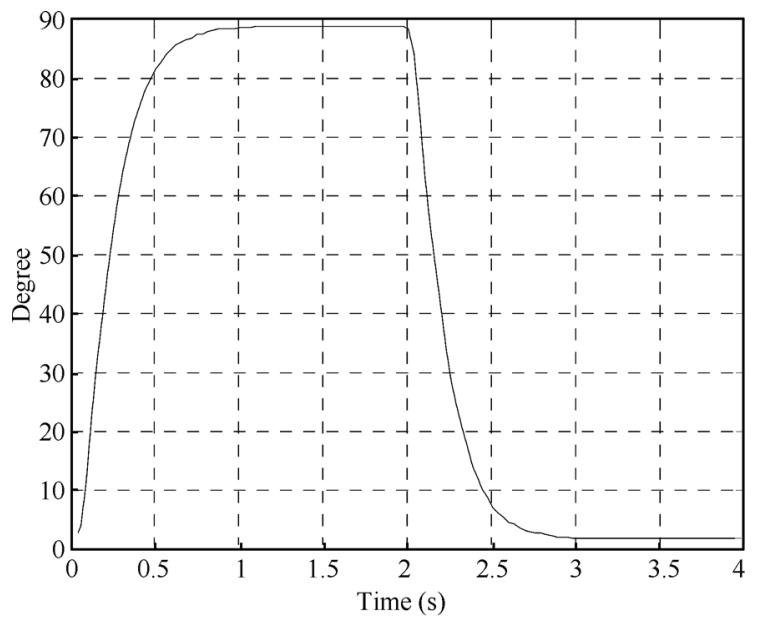

(a) Motor position trajectory at the 1 st iteration

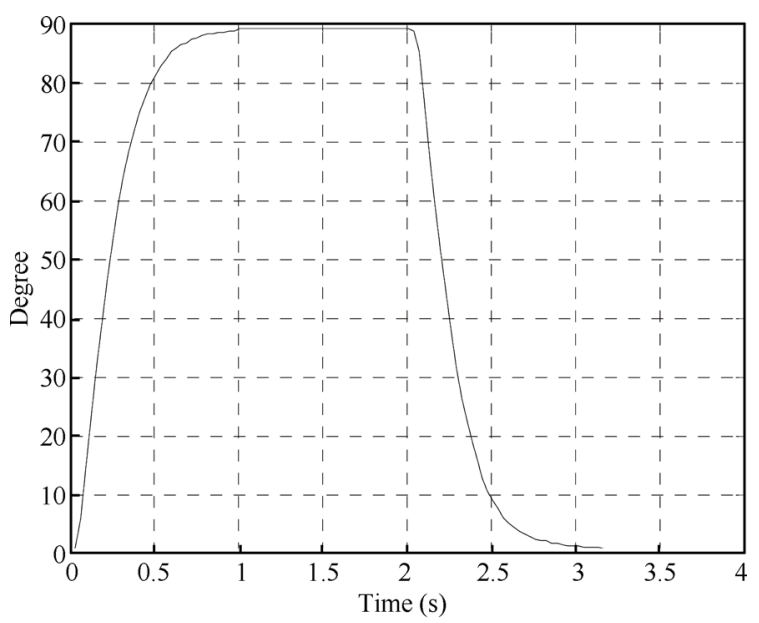

(b) Motor position trajectory at the 5th iteration

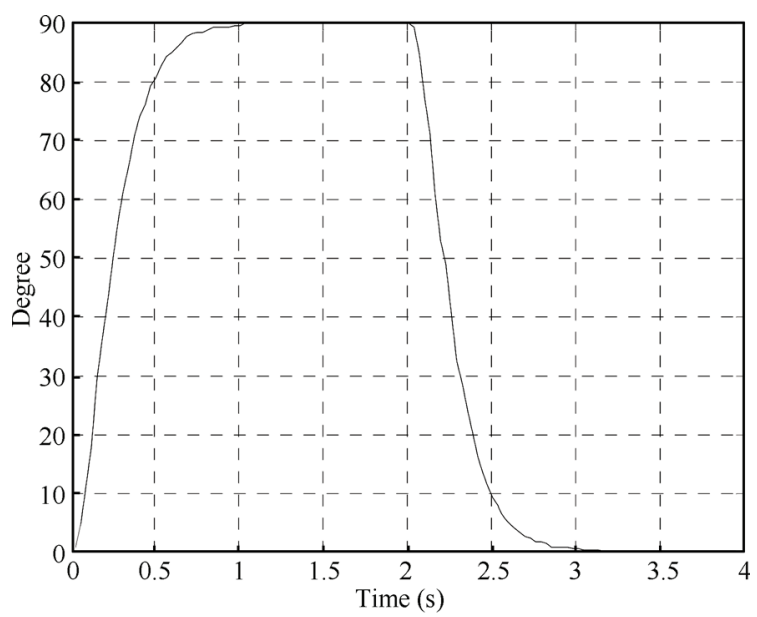

(c) Motor postion trajectory at the 10th iteration

Fig. 6 Motor position trajectory

\section{References}

[1] D. A. Bristow, M. Tharayil, A. G. Alleyne. A survey of iterative learning control. IEEE Control Systems Magazine, vol. 26, no. 3, pp. 96-114, 2006. 
[2] H. S. Ahn, Y. Chen, K. L. Moore. Iterative learning control: Brief survey and categorization. IEEE Transactions on Systems, Man, and Cybernetics, Part C: Applications and Reviews, vol. 37, no. 6, pp. 1099-1121, 2007.

[3] S. S. Saab. On the P-type learning control. IEEE Transactions on Automatic Control, vol. 39, no. 11, pp. 2298-2302, 1994.

[4] K. H. Park, Z. Bien, D. H. Hwang. A study on the robustness of a PID-type iterative learning controller against initial state error. International Journal of Systems Science, vol. 30, no. 1, pp. 49-59, 1999.

[5] Y. Chen, C. Wen, Z. Gong, M. Sun. An iterative learning controller with initial state learning. IEEE Transactions on Automatic Control, vol. 44, no. 2, pp.371-376, 1999.

[6] M. X. Sun, D. W. Wang. Iterative learning control with initial rectifying action. Automatica, vol. 38 , no. 7 , pp. $1177-$ 1182, 2002

[7] J. X. Xu, Z. H. Qu. Robust iterative learning control for a class of nonlinear systems. Automatica, vol.34, no. 8, pp. 983-988, 1998.

[8] J. X. Xu, B. Viswanathan, Z. H. Qu. Robust learning control for robotic manipulators with an extension to a class of non-linear systems. International Journal of Control, vol. 73, no. 10, pp. 858-870, 2000.

[9] A. Tayebi, C. J. Chien. A unified adaptive iterative learning control framework for uncertain nonlinear systems. IEEE Transactions on Automatic Control, vol.52, no. 10, pp. 1907-1913, 2007.

[10] R. H. Chi, Z. S. Hou, J. X. Xu. Adaptive ILC for a class of discrete-time systems with iteration- varying trajectory and random initial condition. Automatica, vol.44, no. 8 , pp. 2207-2213, 2008 .

[11] W. S. Chen, R. H. Li, J. Li. Observer-based adaptive iterative learning control for nonlinear systems with timevarying delays. International Journal of Automation and Computing, vol. 7, no. 4, pp.438-446, 2010.

[12] C. J. Chien. A combined adaptive law for fuzzy iterative learning control of nonlinear systems with varying control tasks. IEEE Transactions on Fuzzy Systems, vol.16, no. 1, pp. 40-51, 2008.

[13] S. Gunnarsson, M. Norrlöf. On the design of ILC algorithms using optimization. Automatica, vol.37, no. 12, pp. 20112016, 2001

[14] K. L. Barton, A. G. Alleyne. A norm optimal approach to time-varying ILC with application to a multi-axis robotic testbed. IEEE Transactions on Control Systems Technology, vol. 19, no. 1, pp. 166-180, 2010.
[15] J. E. Kurek, M. B. Zaremba. Iterative learning control synthesis based on 2-D system theory. IEEE Transactions on Automatic Control, vol. 38, no. 1, pp. 121-125, 1993.

[16] S. S. Saab. A discrete-time learning control algorithm for a class of linear time-invariant systems. IEEE Transactions on Automatic Control, vol. 40, no. 6, pp. 1138-1142, 1995.

[17] J. X. Xu. Analysis of iterative learning control for a class of nonlinear discrete-time systems. Automatica, vol. 33 no. 10, pp. 1905-1907, 1997.

[18] D. Wang. Convergence and robustness of discrete time nonlinear systems with iterative learning control. Automatica, vol. 34, no. 11, pp. 1445-1448, 1998.

[19] S. K. Tso, L. Y. X. Ma. Discrete learning control for robots: Strategy, convergence and robustness. International Journal of Control, vol. 57, no. 2, pp. 273-291, 1993.

[20] C. J. Chien. A sampled-data iterative learning control using fuzzy network design. International Journal of Control, vol. 73, no. 10, pp. 902-913, 2000.

[21] M. X. Sun, D. W. Wang. Sampled-data iterative learning control for nonlinear systems with arbitrary relative degree. Automatica, vol.37, no. 2, pp. 283-289, 2001.

[22] T. D. Son, H. S. Ahn, K. L. Moore. Iterative learning control in optimal tracking problems with specified data points. Automatica, vol. 49, no. 5, pp. 1465-1472, 2013.

[23] Z. S. Hou, Y. Wang, C. K. Yin, T. Tang. Terminal iterative learning control based station stop control of a train. International Journal of Control, vol.84, no. 7, pp. 1263-1274, 2011.

[24] R. H. Chi, D. W. Wang, Z. S. Hou, S. Jin. Data-driven optimal terminal iterative learning control. Journal of Process Control, vol. 22, no. 10, pp. 2026-2037, 2012.

[25] C. J. Chien. A discrete iterative learning control for a class of nonlinear time-varying systems. IEEE Transactions on Automatic Control, vol. 43, no. 5, pp. 748-752, 1998.

[26] Z. S. Hou, J. X. Xu. A new feedback-feedforward configuration for the iterative learning control of a class of discrete-time systems. Acta Automatica Sinica, vol. 33, no. 3, pp. 323-326, 2007.

[27] P. R. Ouyang, B. B. A. Petz, F. F. Xi. Iterative learning control with switching gain feedback for nonlinear systems. Journal of Computational and Nonlinear Dynamics, vol. 6, no. 1, Article number. 011020, 2010.

[28] P. R. Ouyang. PD-PD type iterative learning control for time-varying uncertain nonlinear systems. Control and Intelligent Systems, vol. 39, no. 2, pp. 98-107, 2011. 
[29] C. J. Chien, J. S. Liu. A P-type iterative learning controller for robust output tracking of nonlinear time-varying systems. International Journal of Control, vol.64, no. 2, pp. 319-334, 1996.

[30] Y. Q. Chen, J. X. Xu, T. H. Lee. High-order iterative learning control of discrete-time nonlinear systems using current iteration tracking error. Analysis, Design, Integration and Applications, Iterative Learning Control. USA: Kluwer Academic Publishers, pp. 83-103, 1998.

[31] C. J. Chien, Y. C. Hung, R. H. Chi. Design and analysis of current error based sampled-data ILC with application to position tracking control of DC motors. In Proceedings of the 11th IEEE International Conference on Control \& Automation, IEEE, Taichung, Taiwan, China, pp. 1162-1167, 2014 .

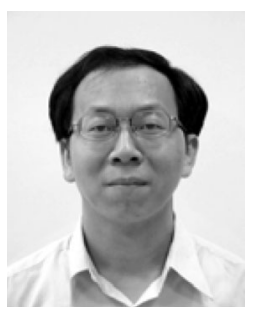

Chiang-Ju Chien received the B. Sc. degree in nuclear engineering from National Tsing Hua University, Taiwan, China in 1985 , and the $\mathrm{Ph} . \mathrm{D}$. degree in electrical engineering from National Taiwan University, Taiwan, China in 1992. Since 1993, he has been with the Department of Electronic Engineering, Huafan University, Taiwan, China, where he is currently a professor. He served as the chair of the same department from 2000 to 2005 and the secretary general of secretariat from 2005 to 2011 in Huafan University. He has been the dean of College of Engineering and Management, Huafan University since 2012. He is a member of IEEE and the Chinese Automatic Control Society.
His research interests include iterative learning control, adaptive control, fuzzy-neuro systems, and control circuit design.

E-mail: cjc@cc.hfu.edu.tw (Corresponding author)

ORCID iD: 0000-0001-8672-0014

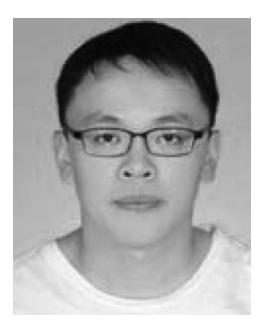

Yu-Chung Hung received the B. Sc. and M. Sc. degrees in electronic engineering from Huafan University, Taiwan, China in 2012 and 2014 respectively.

His research interests include iterative learning control and digital circuit design.

E-mail: lineagezear@yahoo.com.tw

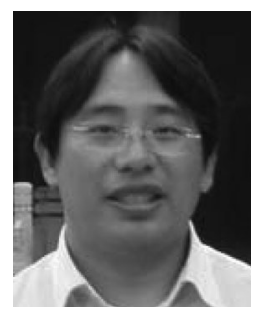

Rong-Hu Chi received the B.Sc. and M. Sc. degrees from Northwest A\&F University, China in 1998 and 2003, respectively, and the Ph. D. degree from Beijing Jiaotong University, China in 2007. He was a visiting scholar with Nanyang Technological University, Singapore from November 2011 to May 2012. In 2007, he joined Qingdao University of Science and Technology, China. He is currently an associate professor in the School of Automation and Electronic Engineering, Qingdao University of Science and Technology.

His research interests include iterative learning control, adaptive control, data-driven control, model-free adaptive control, and intelligent transportation systems.

E-mail: ronghu_chi@hotmail.com 